\title{
Surface-based shared and distinct resting functional connectivity in attention-deficit hyperactivity disorder and autism spectrum disorder
}

Minyoung Jung, Yiheng Tu, Joel Park, Kristen Jorgenson, Courtney Lang, Wenwen Song and Jian Kong

\section{Background}

Both attention-deficit hyperactivity disorder (ADHD) and autism spectrum disorder (ASD) are neurodevelopmental disorders with a high prevalence. They are often comorbid and both exhibit abnormalities in sustained attention, yet common and distinct neural patterns of ASD and ADHD remain unidentified.

\section{Aims}

To investigate shared and distinct functional connectivity patterns in a relatively large sample of boys (7- to 15-year-olds) with ADHD, ASD and typical development matched by age, gender and IQ.

\section{Method}

We applied machine learning techniques to investigate patterns of surface-based brain resting-state connectivity in 86 boys with ASD, 83 boys with ADHD and 125 boys with typical development.

\section{Results}

We observed increased functional connectivity within the limbic and somatomotor networks in boys with ASD compared with boys with typical development. We also observed increased functional connectivity within the limbic, visual, default mode, somatomotor, dorsal attention, frontoparietal and ventral attention networks in boys with ADHD compared with boys with ASD. In addition, using a machine learning approach, we were able to discriminate typical development from ASD, typical development from ADHD and ASD from ADHD with accuracy rates of $76.3 \%, 84.1 \%$, and $79.3 \%$, respectively.

\section{Conclusions}

Our results may shed new light on the underlying mechanisms of ASD and ADHD and facilitate the development of new diagnostic methods for these disorders.

\section{Declaration of interest}

J.K. holds equity in a startup company, MNT.

\section{Keywords}

Developmental disorders; imaging; neuropathology.

\section{Copyright and usage}

(c) The Royal College of Psychiatrists 2018.
Attention-deficit hyperactivity disorder (ADHD) is a neurodeve lopmental disorder characterised by inattention and abnormal hyperactivity and impulsivity, affecting nearly $6 \%$ of children. ${ }^{1}$ Autism spectrum disorder (ASD) is another highly prevalent neurodevelopmental disorder characterised by difficulties in social communication and social interaction. ${ }^{2}$ ADHD and ASD are often comorbid, ${ }^{3,4}$ with about $30 \%$ of patients with ASD having comorbid ADHD characterised by age-inappropriate inattention, impulsiveness and hyperactivity. Neuroimaging studies have shown that both disorders are associated with abnormal restingstate functional brain connectivity. ${ }^{5-12}$ For instance, studies have suggested that compared with typical development, (a) children with ADHD showed disrupted functional connectivity patterns in brain regions involved in attention and sensory processing ${ }^{7,8}$ and (b) children with ASD displayed increased resting-state functional connectivity in the posterior cingulate cortex and salience network, and the strength of functional connectivity was linked to severity of social interaction deficits. ${ }^{5,9,12}$ These studies have significantly enhanced the neurophysiological understanding of ADHD and ASD. Nevertheless, the mechanisms underlying the comorbidity and distinction between the two disorders remain unclear.

In this study, taking advantage of the Autism Brain Imaging Data Exchange (ABIDE) and ADHD200 data-set (http://fcon_1000. projects.nitrc.org/indi/adhd200/), we investigated shared and distinct functional connectivity patterns in a relatively large sample of boys (7- to 15-year-olds) with ADHD, ASD and typical development matched by age, gender and IQ. For better functional alignment across participants, the FreeSurfer image analysis suite was applied to generate a cortical surface for each participant. ${ }^{13} \mathrm{We}$ hypothesised that (a) children with ASD and ADHD would be associated with altered functional connectivity compared with children with typical development, and the altered patterns may be associated with symptoms of ASD and ADHD; and (b) machine learning techniques could be used to identify distinct and common functional connectivity features for both ASD and ADHD.

\section{Method}

\section{Participants}

We used an independent sample of individuals with ASD and individuals with typical development from ABIDE and individuals with ADHD from the ADHD200 data-set (http://fcon_1000.projects. nitrc.org/indi/adhd200/). The inclusion criteria were as follows: (a) full-scale IQ (F-IQ) scores $>80$; (b) aged between 7 and 15 years to minimise potential developmental effects; ${ }^{14}$ (c) scanned in a $3 \mathrm{~T}$ magnetic resonance imaging (MRI) scanner to increase between-site reliability; ${ }^{15}$ (d) right-handed; (e) diagnosis of ASD based on DSMIV-TR ${ }^{16}$ and assessed with the Autism Diagnostic Observation Schedule, ${ }^{17}$ the Autism Diagnostic Interview-Revised (ADI-R), ${ }^{18}$ or both; (f) children with ASD do not have comorbid ADHD and children with ADHD do not have comorbid ASD based on the data-set; (g) diagnosis of ADHD based on DSM-IV-TR without Axis I disorders. Individuals with ASD completed the Social Responsiveness Scale (SRS), a 65-item rating measure that quantifies severity of ASD. ${ }^{19}$ In total, 294 participants fitted the above criteria and were included in the present study. 
In total, 55 children with ASD (64\%), 61 children with ADHD (73\%) and all children with typical development were psychotropic medication-naive. Among children with ADHD, 44 met criteria for combined type ADHD, 3 met criteria for hyperactive/impulsive type $\mathrm{ADHD}$ and 36 met criteria for predominantly inattentive type ADHD. Each site was required to confirm that their local Institutional Review Board or ethics committee had approved both the initial data collection and sharing the data-sets. Details of site-specific protocols, informed consent, and ethical approval at the time of the scan for each data-set can be found at http://fcon_1000.projects.nitrc.org.

\section{Data preprocessing}

Anatomical image data were processed using FreeSurfer, version 5.3.0 software package (http://surfer.nmr.mgh.harvard.edu/). ${ }^{20}$ To increase anatomical validation across individuals with ADHD, ASD, and typical development, FreeSurfer was used for segmentation of subcortical structures and automatic tessellation of the cortical surface because cortical surface variability is considerably improved by segmentation. The preprocessing of anatomical data was as follows: (a) motion correction and non-uniformity correction, (b) automatic Talairach transformation, (c) intensity normalisation, (d) skull strip and segmentation of the subcortical white matter and grey matter, (e) tessellation of the white matter and grey matter, (f) surface smoothing and inflation, (g) topology correction and $(\mathrm{h})$ parcellation. Automated segmentation and parcellation results were reviewed for quality and corrected by two trained experts (M.J. and W.S.) as necessary.

Resting-state functional MRI (fMRI) data-sets were processed with the CONN functional connectivity toolbox (http://www.nitrc. org/projects/conn). ${ }^{21}$ Preprocessing involved (a) realignment to the mean image, (b) removal of volumes with a mean intensity $>1.5 \%$ of the mean global signal or $0.5 \mathrm{~mm} /$ repetition rate framewise displacement to reduce the effect of head movement, (d) CompCor correction to reduce physiological and other noise artefacts, ${ }^{22}$ (e) entering segmented cerebrospinal fluid and white matter as confounding regressors at the participant-level in FreeSurfer, and (f) band-pass filtering of the functional image $(0.01-0.08 \mathrm{~Hz})$.

\section{Regions of interest (ROIS) and connectivity analysis}

We used 162 ROIs adopted from the Desikan-Killiany parcellation atlas in FreeSurfer ${ }^{23}$ (supplementary Table 1 and supplementary Fig. 1 available at https://doi.org/10.1192/bjp.2018.248). Mean time series were obtained for each participant by averaging the fMRI time series over all voxels in each of the 162 ROIs. Functional connectivity was estimated based on these regional mean time series by calculating the pairwise Pearson correlation coefficient between all possible $(162 \times 161 / 2=13041)$ ROI pairs. A symmetric connectivity matrix was constructed to represent these connections. Correlation coefficients were Fisher $z$-transformed to increase normality for statistical analyses.

\section{Classification analysis: discriminating typical development, ASD and ADHD}

To investigate diagnostic features between ADHD and ASD, a feature-selection approach combining a univariate $t$-test and multivariate support vector machine-recursive feature elimination (SVM-RFE) was performed. ${ }^{24}$ To avoid the risk of overfitting, all analyses were performed using ten-fold cross-validation. ${ }^{25}$

In the first step, we analysed group-level differences of features between groups. Significant differences for each pair of ROIs were assessed using a mass univariate two sample $t$-test with a threshold of $P<0.001$ and false discovery rate (FDR) correction. Features showing significant difference were retained for the remaining analyses. Our logic was that these features would be the most likely to contribute to the discrimination between groups.

In the second step, we used SVM-RFE to select the features with the most discriminative power for the classifier itself. SVM-RFE was used to train the classification model and obtain weights for each feature. The features were ranked according to the absolute values of weights, and the lowest ranking feature was discarded. Then the classification model was trained using the new feature set (i.e. without the discarded feature). This procedure was repeatedly performed until the feature set was empty. We conducted a full backward elimination procedure to further select the features with the highest classification accuracy. Since we used a ten-fold cross-validation strategy to estimate the performance of the classifiers and feature ranking and each iteration was based on a slightly different data-set, the selected feature sets differed slightly from iteration to iteration.

To determine the most discriminative features, a consensus discrimination map that aggregated features selected in all cross-validation iterations was used. Regional weight, which represents the contribution of each feature for discriminating different groups, was denoted by the number of ROI occurrences in the consensus discrimination map. ${ }^{26}$ The discriminative power of each feature was denoted by the average of its classification weights across all iterations. We conducted linear regression analyses of group-level differences to select features based on SVM-RFE and core symptom severity in ASD (total SRS scores), adjusting for data-collection site, F-IQ and age using SPSS. Regression analyses were corrected for multiple comparisons using FDR correction $(P<0.05)$.

Features with the most discriminative power were fed to an SVM with a linear kernel, which was implemented using LIBSVM. The classification (ASD versus typical development; ADHD versus typical development; ADHD versus ASD) was also based on ten-fold cross-validation, and the performance of the classifier was evaluated by accuracy, sensitivity and specificity. Non-parametric permutation tests (1000 times) were used to estimate the statistical significance of the observed classification accuracy. We randomly permuted the class labels of the data prior to training. Cross-validation was then performed on the permuted data-set and the procedure was repeated 1000 times. If a classifier trained on real class labels had an accuracy exceeding the $95 \%$ confidence interval generated from the accuracies of the classifiers trained on randomly relabelled class labels, this classifier was considered to be well-performing.

\section{Results}

\section{Demographic and clinical characteristics}

In total, 294 participants ( 86 boys with ASD, 83 boys with ADHD and 125 boys with typical development) were included in the study. There were no significant differences between the three groups for F-IQ $(P=0.51)$ and age $(P=0.27)$. Demographic and clinical characteristics for all participants included in the analyses are presented in Table 1. There was a significant difference between ASD and typical development groups for SRS total scores $(P<0.0001)$.

\section{Diagnostic features of ROI-to-ROI functional connectivity analysis}

Comparison of the typical development and ASD groups showed 72 ROI-to-ROI increased connectivities in the ASD group compared with the typical development group (Fig. 1). The ASD group showed increased connectivity in brain areas associated with the limbic, visual, default mode, somatomotor, dorsal attention, frontoparietal and ventral attention networks. The typical development group only showed nine ROI-to-ROI increased connectivities in brain areas associated with the visual, default mode, dorsal 


\begin{tabular}{|c|c|c|c|c|c|c|c|c|c|}
\hline \multirow[b]{2}{*}{ Measure } & \multicolumn{2}{|c|}{$\begin{array}{l}\text { Autism spectrum } \\
\text { disorder }(n=86)\end{array}$} & \multicolumn{2}{|c|}{$\begin{array}{c}\text { Typical } \\
\text { development group } \\
(n=125) \\
\end{array}$} & \multicolumn{2}{|c|}{$\begin{array}{l}\text { Attention-deficit } \\
\text { hyperactivity } \\
\text { disorder group } \\
(n=83)\end{array}$} & \multicolumn{3}{|c|}{ Group comparisons } \\
\hline & Mean & s.d. & Mean & s.d. & Mean & s.d. & $F$-value & $t$-test & $P$ \\
\hline Age (years) & 11.4 & 2.1 & 10.9 & 1.6 & 11.2 & 1.8 & 1.32 & - & 0.267 \\
\hline Full IQ & 110.4 & 13.2 & 111.7 & 9.7 & 109.9 & 13.1 & 0.67 & - & 0.512 \\
\hline \multicolumn{10}{|l|}{ ADI-R } \\
\hline Social & 18.9 & 4.9 & - & - & - & - & - & - & - \\
\hline Non-verbal & 8.34 & 3.1 & - & - & - & - & - & - & - \\
\hline Verbal & 14.9 & 4.3 & - & - & - & - & - & - & - \\
\hline SRS total scores & 91.8 & 25.7 & 19.2 & 12.4 & - & - & - & 26.3 & $<0.0001$ \\
\hline
\end{tabular}

attention, frontoparietal and ventral attention networks compared with the ASD group. The classification accuracy of discriminating typical development from ASD was $76.3 \%(P<0.001$; Table 2$)$.

Comparison of the typical development and ADHD groups showed eight ROI-to-ROI increased connectivities in the ADHD group compared with the typical development group (Fig. 2). Specifically, the ADHD group showed increased connectivity in brain areas associated with the limbic, ventral attention, visual and default mode networks. In addition, the typical development group showed two ROI-to-ROI increased connectivities in brain areas associated with the default mode and visual networks compared with the ADHD group. The classification accuracy of discriminating typical development from ADHD was $84.1 \%(P<0.001$; Table 2$)$.

Comparison of the ASD and ADHD groups showed nine ROI-toROI increased connectivities in the ADHD group compared with the ASD group (supplementary Fig. 2). Specifically, the ADHD group showed increased functional connectivity between brain regions associated with the limbic, visual, default mode, somatomotor, dorsal attention, frontoparietal and ventral attention networks. In addition, we found that the ASD group showed increased functional connectivity in the left middle occipital sulcus and right precentral sulcus associated with the somatomotor and dorsal attention networks compared with the ADHD group. The classification accuracy of discriminating ADHD from ASD was $79.3 \%(P<0.001$; Table 2$)$.

\section{Association between functional connectivity and core symptom severity}

Regression analysis showed a positive association between increased connectivity in the ASD group compared with the typical development group and SRS scores, specifically (a) with the right insula and post transverse collateral sulcus functional connectivity $(P=0.0004, P=0.01$ FDR corrected; $r=0.376)$ and $(b)$ with the right orbital gyrus and right horizontal ramus of the lateral sulcus functional connectivity ( $P=0.0076, P=0.025$ FDR corrected; $r=$ 0.309 ) (supplementary Fig. 3 and supplementary Table 2).

\section{Discussion}

\section{Main findings}

In this study, we investigated shared and distinct patterns of functional dysconnectivity in boys with ASD and ADHD. We found that (a) children with ASD showed increased functional connectivity compared with children with typical development, and children with ADHD showed increased functional connectivity compared with children with ASD and children with typical development; and (b) machine learning approaches can discriminate ASD and ADHD with accuracies of: $76.3 \%$ (ASD from typical development), 84.1\% (ADHD from typical development) and 79.3\% (ADHD from ASD). Our results may deepen our understanding of the neurophysiological mechanisms underlying the comorbidity and distinction between ADHD and ASD.

\section{Comparison with findings from other studies}

Our findings of increased functional connectivity patterns within the limbic regions and the somatomotor network in ASD compared with typical development are consistent with previous studies. ${ }^{12}$ For
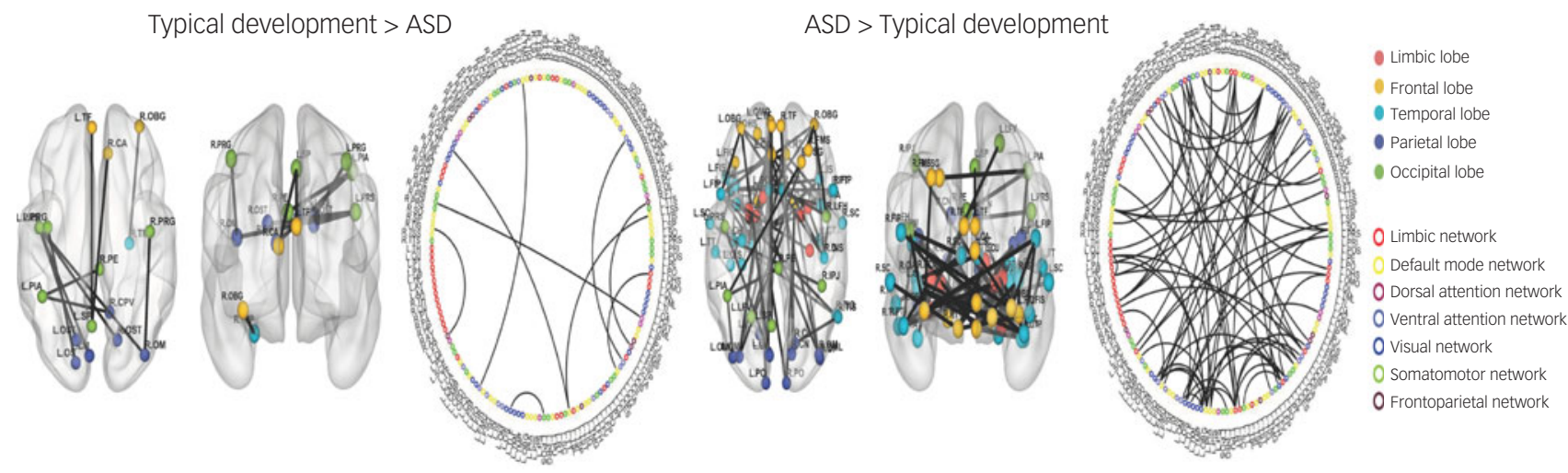

Fig. 1 Region of interest (ROI)-to-ROI functional connectivity analysis between typical development and autism spectrum disorder (ASD).

The ASD group showed predominantly higher connectivity than the typical development group. Coloured circles indicate brain regions. Coloured lines indicate networks. L., left; R., right; see supplementary Table 1 for details of the other abbreviations used in this figure. 


\begin{tabular}{lcccc} 
Table 2 Results of machine learning analysis & & & \multicolumn{2}{c}{ Positive likelihood } \\
ratio & Accuracy & Sensitivity & Specificity & Negative likelihood ratio \\
Method & 76.3 & 79.2 & 63.9 & 2.19 \\
Typical development_autism spectrum disorder & 84.1 & 88.8 & 76.1 & 3.72 \\
Typical development_attention-deficit hyperactivity disorder & 79.3 & 75.6 & 83.1 & 0.33 \\
Autism spectrum disorder_attention-deficit hyperactivity & & & & 0.15 \\
$\quad$ disorder & &
\end{tabular}

instance, Cerliani and colleagues found increased functional connectivity in the limbic area and sensory-motor area in male patients with ASD. ${ }^{12}$ Likewise, the DSM-5 manual also includes abnormal responses to sensory stimulation as a diagnostic criterion of ASD. Our results suggest that increased functional connectivity in the limbic and sensory-motor areas might reflect this abnormal response. The finding of increased functional connectivity patterns in children with ADHD compared with typical development is also consistent with previous studies on ADHD. ${ }^{6}$ Studies have suggested that the default network and limbic area are associated with regulation of attention, ${ }^{27}$ self-cognition ${ }^{28}$ and external cognition. ${ }^{29}$ We thus speculate that increased functional connectivity may underlie impairments in 'sharing attention with other people,' which is a core symptom of ADHD.

We also found increased functional connectivity in the frontal lobe, temporal lobe, occipital lobe, parietal lobes, frontoparietal network and ventral attention network in the ADHD group compared with the typical development group. Rubia et al found that temporal lobe and parietal lobe dysfunction in boys with ADHD during an attention allocation task was associated with symptoms of ADHD. ${ }^{30}$ We believe that abnormal functional connectivity in the temporal lobe and parietal lobe might disrupt or delay maturation of the regulation of attention in ADHD.

Nonetheless, our findings are inconsistent with other studies indicating that ADHD is related to decreased functional connectivity in the posterior cingulate cortex ${ }^{7}$ and increased functional connectivity within frontal regions of the executive control network ${ }^{8}$ as compared with typical development. These conflicting findings may be the result of inconsistent methodologies or variability in the ADHD sample population. For instance, Kyeong et al used graph theory analysis to estimate degree centrality in stratifying ADHD subgroups with mild symptom ADHD and severe symptom ADHD. Francx et $a l^{8}$ used independent component analysis to detect components or networks in persistent ADHD subgroups and remittent $\mathrm{ADHD}$ subgroups. These discrepancies illustrate the importance of methodology and clinical subgroup differences during the interpretation of neuroimaging study findings.

\section{Interpretation of our findings}

We found that children with ASD showed increased functional connectivity between the left middle occipital sulcus and right precentral sulcus compared with ADHD. The occipital lobe and parietal lobe are involved in communication processing, including emotion perception ${ }^{31}$ and face discrimination, ${ }^{32}$ as well as the pathophysiology of autism. ${ }^{33}$ A previous study indicated that ASD involves a different cognitive process during social interactions. ${ }^{34}$ We speculate that this may be because of the increased functional connectivity between the occipital and parietal lobes compared with ADHD and typical development. These results may provide an explanation for the altered communication processing at the neural level in individuals with ASD.

Our machine-learning algorithms confirmed common classification features between ASD and ADHD in the limbic, ventral attention, visual and default mode networks. This finding is consistent with previous studies that found abnormal functional connectivity in these networks in both ASD and ADHD. ${ }^{5,6,35}$ Recent studies indicate that ADHD may be associated with difficulties in social interaction. ${ }^{36}$ Symptoms of ADHD (e.g. attention deficits, impulse control and hyperactivity) are also frequently observed in ASD, demonstrating that the two disorders share some common manifestations. Taken together, the common classification features between ASD and ADHD may reflect shared neural mechanisms and clinical manifestations in the two disorders.

Translational neuroimaging studies have provided a basis for identifying neurophysiological features of ASD and showed potential clinical utility. ${ }^{35}$ Specifically, advanced machine learning techniques have been introduced to extract meaningful features from neuroimaging data and subsequently make an objective diagnosis for ASD. Anderson and colleagues used univariate $t$-tests to
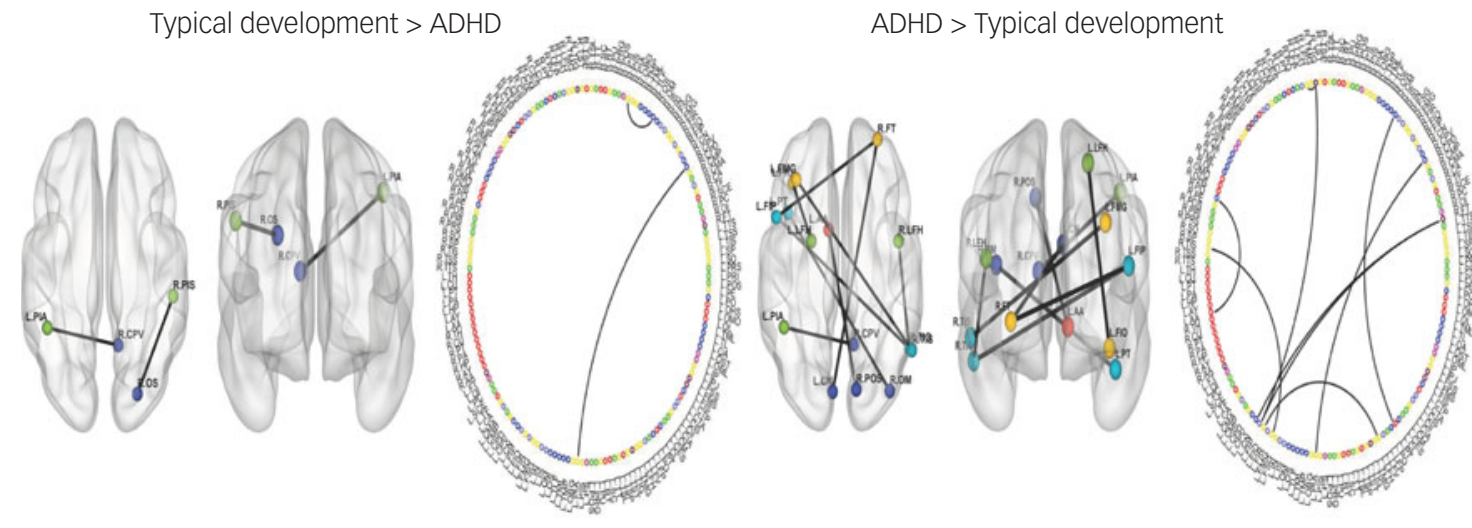

- Limbic lobe

Frontal lobe

Temporal lobe

- Parietal lobe

occipital lobe

O Limbic network

Default mode network

O Dorsal attention network

$O$ ventral attention network

0 visual network

O Somatomotor network

O Frontoparietal network

Fig. 2 Region of interest (ROI)-to-ROI functional connectivity analysis between typical development and attention-deficit hyperactivity disorder (ADHD).

The ADHD group showed predominantly higher connectivity than the typical development group. Coloured circles indicate brain regions. Coloured lines indicate networks. L., left; R., right; see supplementary Table 1 for details of the other abbreviations used in this figure. 
exclude irrelevant functional connectivities and achieved an accuracy of $79 \% .{ }^{37}$ Nielsen et al used a leave-one-out classifier with a general linear model on the multisite ABIDE data-set and obtained accuracies of up to $60 \%$ for different sites. ${ }^{38}$

More recently, Yahata and his colleagues developed a machine learning algorithm combining $L_{1}$-regularised sparse canonical correlation analysis and sparse logistic regression for selecting a subset of functional connectivities to obtain a classification accuracy of around $85 \%$ in a Japanese data-set, but generalisation for independent cohorts using two independent validation cohorts obtained from the ABIDE data-set showed lower accuracy (75\%). ${ }^{39}$ In the present study, we combined univariate $t$-tests and multivariate SVM-RFE to identify the most discriminative features between ASD, ADHD and typical development using seven independent cohorts and obtained accuracies of $76.3 \%$ between typical development and ASD, 84.1\% between typical development and ADHD, and $79.3 \%$ between ASD and ADHD. Our results demonstrated that a classifier developed using surface-based functional connectivity also showed high classification for ASD and ADHD across other independent cohorts.

We found that the increased functional connectivity in the right insula and right orbital cortex was associated with SRS scores. Functional and structural imaging studies of ASD have identified abnormalities in the insula, explaining the emotion dysregulation and social avoidance symptoms of ASD. ${ }^{40,41}$ The orbital cortex, a critical brain region in social cognition, has been associated with high levels of autistic traits. ${ }^{42}$ Taken together, these results suggest that atypical connectivity in the insula and orbital cortex are related to emotion dysregulation and social cognition.

\section{Limitations}

There are several limitations in this study. First, our analyses were performed on boys with ASD who did not have comorbid ADHD and boys with ADHD who did not have comorbid ASD based on the data-set. Yet, we did not have ADHD symptom scores for participants with ASD or ASD scores for participants with ADHD. We thus cannot exclude the possibility that the disorders were comorbid. Further research including both ASD and ADHD symptom scores is needed. Second, this study only included boys with typical development, ASD and ADHD; thus, the results may not be generalisable to girls. Future studies including both boys and girls with ASD and ADHD are needed.

\section{Implications}

In summary, we found that boys with ASD are associated with increased functional connectivity in the limbic area, while boys with ADHD are associated with increased functional connectivity in the frontal and temporal areas. Machine learning-derived classification methods hold the potential to uncover neuroimaging biomarkers for ASD and ADHD.

Minyoung Jung (iD, PhD, Assistant Professor, Research Center for Child Mental Development, University of Fukui, Japan; Yiheng Tu, PhD, Research Fellow, Department of Psychiatry, Massachusetts General Hospital, Harvard Medical School, USA; Joel Park BA, Research Coordinator, Department of Psychiatry, Massachusetts General Hospital, Harvard Medical School, USA; Kristen Jorgenson, BA, Research Coordinator, Department of Psychiatry, Massachusetts General Hospital, Harvard Medical School, USA; Courtney Lang, BA, Research Coordinator, Department of Psychiatry, Massachusetts General Hospital, Harvard Medical School, USA; Wenwen Song, MD, Radiologist, The First Affiliated Hospital of Zhejiang Chinese Medical University, China; Jian Kong, MD, MS, MPH, Associated Professor, Department of Psychiatry, Massachusetts General Hospital, Harvard Medical School, USA

Correspondence: Jian Kong, MD, MS, MPH, Department of Psychiatry, Massachusetts General Hospital, Harvard Medical School, Charlestown, MA 02129, USA. Email: kongj@ nmr.mgh.harvard.edu

First received 25 Jan 2018, final revision 28 Aug 2018, accepted 14 Oct 2018

\section{Supplementary material}

Supplementary material is available online at https://doi.org/10.1192/bjp.2018.248.

\section{Funding}

Minyoung Jung is supported by 18 K13106 from the Japanese Ministry of Education, Culture, Sports, Science, and Technology (MEXT) of Japan, Jian Kong is supported by R01 AT008563, R21AT008707, and R33AT009310 from NIH/NCCIH.

\section{References}

1 Moffitt TE, Houts R, Asherson P, Belsky DW, Corcoran DL, Hammerle M, et al. Is adult ADHD a childhood-onset neurodevelopmental disorder? Evidence from a four-decade longitudinal cohort study. Am J Psychiatry 2015; 172: 967-77.

2 Lai M-C, Lombardo M V, Baron-Cohen S. Autism. Lancet 2014; 383: 896-910.

3 Simonoff E, Pickles A, Charman T, Chandler S, Loucas T, Baird G. Psychiatric disorders in children with autism spectrum disorders: prevalence, comorbidity, and associated factors in a population-derived sample. J Am Acad Child Adolesc Psychiatry 2008; 47: 921-9.

4 Park B, Hong J, Lee S-H, Park H. Functional connectivity of child and adolescent attention deficit hyperactivity disorder patients: correlation with IQ. Front Hum Neurosci 2016; 10: 31-40.

5 Lynch CJ, Uddin LQ, Supekar K, Khouzam A, Phillips J, Menon V. Default mode network in childhood autism: posteromedial cortex heterogeneity and relationship with social deficits. Biol Psychiatry 2013; 74: 212-9.

6 Di Martino A, Zuo X-N, Kelly C, Grzadzinski R, Mennes M, Schvarcz A, et al. Shared and distinct intrinsic functional network centrality in autism and attention-deficit/hyperactivity disorder. Biol Psychiatry 2013; 74: 623-32.

7 Kyeong S, Kim JJ, Kim E. Novel subgroups of attention-deficit/hyperactivity disorder identified by topological data analysis and their functional network modular organizations. PLOS One 2017; 12: e0182603.

8 Francx W, Oldehinkel M, Oosterlaan J, Heslenfeld D, Hartman CA, Hoekstra PJ, et al. The executive control network and symptomatic improvement in attention-deficit/hyperactivity disorder. Cortex 2015; 73: 62-72.

9 Supekar K, Uddin LQ, Khouzam A, Phillips J, Gaillard WD, Kenworthy LE, et al. Brain hyperconnectivity in children with autism and its links to social deficits. Cell Rep 2013; 5: 738-47.

10 Christakou A, Murphy CM, Chantiluke K, Cubillo Al, Smith AB, Giampietro V, et al. Disorder-specific functional abnormalities during sustained attention in youth with attention deficit hyperactivity disorder (ADHD) and with autism. Mol Psychiatry 2013; 18: 236-44.

11 Ray S, Miller M, Karalunas S, Robertson C, Grayson DS, Cary RP, et al. Structural and functional connectivity of the human brain in autism spectrum disorders and attention-deficit/hyperactivity disorder: a rich club-organization study. Hum Brain Mapp 2014; 35: 6032-48.

12 Cerliani L, Mennes M, Thomas RM, Di Martino A, Thioux M, Keysers C. Increased functional connectivity between subcortical and cortical resting-state networks in autism spectrum disorder. JAMA Psychiatry 2015; 72: 1-11.

13 Smith SM, vidaurre D, Beckmann CF, Glasser MF, Jenkinson M, Miller KL, et al. Functional connectomics from resting-state fMRI. Trends Cogn Sci 2013; 17 666-82.

14 Dickstein DP, Pescosolido MF, Reidy BL, Galvan T, Kim KL, Seymour KE, et al Developmental meta-analysis of the functional neural correlates of autism spectrum disorders. J Am Acad Child Adolesc Psychiatry 2013; 52: 279-89.e16.

15 Friedman L, Stern H, Brown GG, Mathalon DH, Turner J, Glover GH, et al. Testretest and between-site reliability in a multicenter fMRI study. Hum Brain Mapp 2008; 29: 958-72.

16 American Psychiatric Association. Diagnostic and Statistical Manual of Mental Disorders (4th edn, revised) (DSM-IV-TR). APA, 2000.

17 Lord C, Rutter M, DiLavore PC, Risi S, Gotham K, Bishop SL. Autism Diagnostic Observation Schedule, Second Edition (ADOS-2) Modules 1-4. Western Psychological Services, 2012

18 Lord C, Rutter M, Le Couteur A. Autism Diagnostic Interview-Revised: a revised version of a diagnostic interview for caregivers of individuals with possible pervasive developmental disorders. J Autism Dev Disord 1994; 24: 659-85.

19 Constantino JN, Davis SA, Todd RD, Schindler MK, Gross MM, Brophy SL, et al. Validation of a brief quantitative measure of autistic traits: Comparison of the social responsiveness scale with the Autism Diagnostic Interview-Revised. J Autism Dev Disord 2003; 33: 427-33.

20 Fischl B, Sereno MI, Tootell RBH, Dale AM. High-resolution intersubject averaging and a coordinate system for the cortical surface. Hum Brain Mapp 1999; 8: $272-84$. 
21 Whitfield-Gabrieli S, Nieto-Castanon A. CONN: a functional connectivity toolbox for correlated and anticorrelated brain networks. Brain Connect 2012; 2: 125-41.

22 Muschelli J, Nebel MB, Caffo BS, Barber AD, Pekar JJ, Mostofsky SH. Reduction of motion-related artifacts in resting state fMRI using aCompCor. Neuroimage 2014; 96: 22-35.

23 Desikan RS, Ségonne F, Fischl B, Quinn BT, Dickerson BC, Blacker D, et al. An automated labeling system for subdividing the human cerebral cortex on MRI scans into gyral based regions of interest. Neuroimage 2006; 31: 968-80.

24 Guyon I, Weston J, Barnhill S, Vapnik V. Gene selection for cancer classification using support vector machines. Mach Learn 2002; 46: 389-422.

25 Tu Y, Zhang Z, Tan A, Peng W, Hung YS, Moayedi M, et al. Alpha and gamma oscillation amplitudes synergistically predict the perception of forthcoming nociceptive stimuli. Hum Brain Mapp 2016; 37: 501-14.

26 Zeng L-L, Shen H, Liu L, Wang L, Li B, Fang P, et al. Identifying major depression using whole-brain functional connectivity: a multivariate pattern analysis. Brain 2012; 135: 1498-507.

27 Leech R, Sharp DJ. The role of the posterior cingulate cortex in cognition and disease. Brain 2014; 137: 12-32.

28 Buckner RL, Andrews-Hanna JR, Schacter DL. The brain's default network: anatomy, function and relevance to disease. Ann N Y Acad Sci 2008; 1124: 1-38.

29 Leech R, Kamourieh S, Beckmann CF, Sharp DJ. Fractionating the default mode network: distinct contributions of the ventral and dorsal posterior cingulate cortex to cognitive control. J Neurosci 2011; 31: 3217-24.

30 Rubia K, Smith AB, Brammer MJ, Taylor E. Temporal lobe dysfunction in medication-naive boys with attention-deficit/hyperactivity disorder during attention allocation and its relation to response variability. Biol Psychiatry 2007; 62: 999-1006.

31 Gschwind M, Pourtois G, Schwartz S, Van De Ville D, Vuilleumier P. Whitematter connectivity between face-responsive regions in the human brain. Cereb Cortex 2012; 22: 1564-76.

32 Jonas J, Rossion B, Krieg J, Koessler L, Colnat-Coulbois S, Vespignani H, et al. Intracerebral electrical stimulation of a face-selective area in the right inferior occipital cortex impairs individual face discrimination. Neuroimage 2014; 99 : 487-97.

33 Jung M, Tu Y, Lang CA, Ortiz A, Park J, Jorgenson K, et al. Decreased structural connectivity and resting-state brain activity in the lateral occipital cortex is associated with social communication deficits in boys with autism spectrum disorder. Neuroimage 2017; Sept 18 : pii S1053-8119(17)30779-6, DOI 10.1016/ j.neuroimage.2017.09.031.

34 Hubbard AL, Mcnealy K, Scott-Van Zeeland AA, Callan DE, Bookheimer SY, Dapretto $M$. Altered integration of speech and gesture in children with autism spectrum disorders. Brain Behav 2012; 2: 606-19.

35 Di Martino A, Kelly C, Grzadzinski R, Zuo XN, Mennes M, Mairena MA, et al. Aberrant striatal functional connectivity in children with autism. Biol Psychiatry 2011; 69: 847-56

36 Hoekzema E, Carmona S, Ramos-Quiroga JA, Richarte Fernández V, Bosch R, Soliva JC, et al. An independent components and functional connectivity analysis of resting state FMRI data points to neural network dysregulation in adult ADHD. Hum Brain Mapp 2014; 35: 1261-72.

37 Anderson A, Douglas PK, Kerr WT, Haynes VS, Yuille AL, Xie J, et al. Nonnegative matrix factorization of multimodal MRI, fMRI and phenotypic data reveals differential changes in default mode subnetworks in ADHD. Neuroimage. 2014; 102: 207-19.

38 Nielsen JA, Zielinski BA, Fletcher PT, Alexander AL, Lange N, Bigler ED, et al. Multisite functional connectivity MRI classification of autism: ABIDE results. Front Hum Neurosci 2013; 7: 599

39 Yahata N, Morimoto J, Hashimoto R, Lisi G, Shibata K, Kawakubo Y, et al. A small number of abnormal brain connections predicts adult autism spectrum disorder. Nat Commun 2016; 7: 11254.

40 Mazefsky CA, Herrington J, Siegel M, Scarpa A, Maddox BB, Scahill L, et al. The role of emotion regulation in autism spectrum disorder. J Am Acad Child Adolesc Psychiatry 2013; 52: 679-88.

41 Kosaka H, Omori M, Munesue T, Ishitobi M, Matsumura Y, Takahashi T, et al. Smaller insula and inferior frontal volumes in young adults with pervasive developmental disorders. Neuroimage 2010; 50: 1357-63.

42 Mayer JL. The relationship between autistic traits and atypical sensory functioning in neurotypical and ASD adults: a spectrum approach. J Autism Dev Disord 2017; 47: 316-27. 